\title{
Recharacterization of Thermal Voltage Converters After Thermoelement Replacement
}

\author{
JOSEPH R. KINARD, SENIOR MEMBER, IEEE, AND THOMAS E. LIPE, MEMBER, IEEE
}

\begin{abstract}
The relationship between the characteristics of various thermoelements (TE's) as voltage or current converters and the overall ac-dc differences of a voltage range in a coaxial thermal voltage converter (TVC) set is described. An algorithm to predict the relationships between the ac-dc differences of individual voltage ranges with different TE's is presented, and a method for recharacterizing a TVC containing a replacement TE is given. The measured results show that for most applications a complete recharacterization of the TVC set is unnecessary.
\end{abstract}

\section{INTRODUCTION}

$\mathrm{T}$ HERMAL voltage converters (TVC's) are often constructed as sets of range resistors and a group of two or more thermoelement (TE) modules. A given voltage range is selected by connecting a TE module in series with an appropriate range resistor, so that the ac-dc differences of a particular range depend on the combined characteristics of the range resistor and the TE. If a thermoelement should fail, the characteristics of a replacement will affect the ac-dc difference of all voltage ranges associated with that TE to some extent.

Analyses of the relationships between the ac-dc difference of the TE and related range resistors for coaxial TVC's have been undertaken by Hermach [1] and Inglis [2]. The analysis presented here is based on that in [1], but explicitly treats phase angle differences in the TE's as well as providing further consideration of loading effects on the series resistor. The model developed here is based on a thermal converter design generally used at frequencies up to $100 \mathrm{kHz}$, and has been tested only up to 100 $\mathrm{kHz}$.

\section{Derivation of Equations}

A coaxial TVC composed of a series resistor and TE in separate modules is shown in Fig. 1. In general the impedance of a module may be related to its dc resistance, at a particular frequency, by in-phase and quadrature variations which are small for well-constructed TVC's. Taking $a$ and $b$, for the series resistor module, and $c$ and $d$, for the TE module, to represent these in-phase and quadrature variations, respectively, the impedances of each

Manuscript received June 10,1988 . This paper was supported in part by the Calibration Coordination Group of the U.S. Department of Defense. The authors are with the Electricity Division, National Bureau of Standards, Gaithersburg, MD 20899.

IEEE Log Number 8825608 .

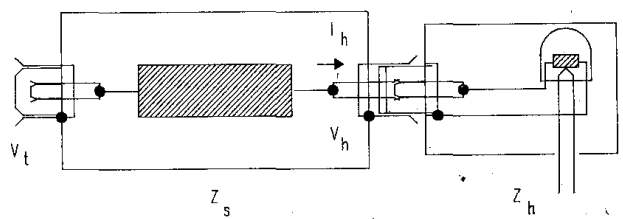

Fig. 1. Schematic of resistor-thermoelement combination showing the transimpedance of the resistor, $Z_{s}$ and the impedance of the TE module, $Z_{h}$

module may be written as

$$
\begin{aligned}
& Z_{s}=R_{s}(1+a+j b) \\
& Z_{h}=R_{h}(1+c+j d)
\end{aligned}
$$

where:

$Z_{s} \quad$ the transimpedance, $\left(V_{t}-V_{h}\right) / I_{h}$, of the series resistor module,

$Z_{h} \quad$ the impedance, $V_{h} / I_{h}$, of the TE module,

$I_{h} \quad$ the input current to the TE module when mated,

$V_{t} \quad$ the TVC input voltage,

$V_{h} \quad$ the voltage at the TE module,

$R_{s} \quad$ the dc resistance of the series resistor module,

$R_{h} \quad$ the dc resistance of the TE module,

$a, b, c$, and $d$ representing the combined effects of whatever ac factors, e.g., reactance, skin effect, dielectric loss, stray capacitance, etc., may contribute to the impedance,

$b$

(approximately) the phase angle, $\theta_{s}$, of the resistor module in radians, since $a$ and $b$ are $\ll 1$ and $\theta_{s}=\tan ^{-1} b /(1$ $+a)$

$d$ (approximately) the phase angle, $\theta_{h}$, of the TE module in radians, since $c$ and $d$ are $\ll 1$ and $\theta_{h}=\tan ^{-1} d /(1+$ c).

Note that in Fig. 1, the outer cases of the modules are electrically continuous, and carry the return current from the low side of the TE back to the input connector.

Letting $p=R_{h} / R_{s}(2)$ becomes

$$
Z_{h}=p R_{s}(1+c+j d) .
$$


The total impedance of the resistor-TE system is then [1]

$$
Z_{t}=Z_{s}+Z_{h}=R_{s}(1+a+j b)+p R_{s}(1+c+j d) \text {. }
$$

The total dc resistance is $R_{t}=R_{s}+R_{h}$. Substituting this quantity into (4) and dividing both sides by $R_{t}$ yields

$$
\frac{Z_{t}}{R_{t}}=\frac{R_{s}(1+a+j b)+p R_{s}(1+c+j d)}{R_{t}} .
$$

Collecting like terms

$$
\frac{Z_{t}}{R_{t}}=1+\frac{a+p c+j(b+p d)}{(1+p)}
$$

Setting $\gamma=(a+p c) /(1+p)$ and $\zeta=(b+p d) /(1+$ $p$ ) and taking the magnitude of $Z_{t}$

$$
\frac{\left|Z_{t}\right|}{R_{t}}=\sqrt{(1+\gamma)^{2}+\zeta^{2}}
$$

Using the binomial expansion for $(x+y)^{1 / 2}$ with $x=(1$ $+\gamma)^{2}$ and $y=\zeta^{2}$ yields, closely enough

$$
\frac{\left|Z_{t}\right|}{R_{t}}=1+\gamma+\frac{1}{2}\left[\frac{1}{1+\gamma}\right] \zeta^{2} .
$$

Since $\gamma$ and $\zeta$ are both $\ll 1,(1 / 1+\gamma) \simeq(1-\gamma)$, and

$$
\frac{\left|Z_{t}\right|}{R_{t}}=1+\gamma+\frac{1}{2} \zeta^{2}-\frac{1}{2} \gamma \zeta^{2}
$$

Neglecting terms higher than second order gives

$$
\frac{\left|Z_{t}\right|}{R_{t}}=1+\gamma+\frac{1}{2} \zeta^{2}
$$

The ac-dc difference of a TVC may be written $\delta_{t}=\left(\left|Z_{t}\right|\right.$ $\left.-R_{t}\right) / R_{t}+\delta_{c}$, where $\delta_{c}$ is its ac-dc difference as a current converter [3]. Substituting (5) into this expression for $\delta_{t}$ gives

$$
\delta_{t}=\gamma+\frac{1}{2} \zeta^{2}+\delta_{c} .
$$

It we consider the case of two TE's (no. 1 and no. 2) of the same nominal, but not identical, heater resistance, the variation in the ac-dc differences caused by the substitution of one TE for the other may be expressed as

$$
\begin{aligned}
\delta_{t_{2}}-\delta_{t_{1}}= & {\left[\frac{a+p_{2} c_{2}}{1+p_{2}}-\frac{a+p_{1} c_{1}}{1+p_{1}}\right]+\frac{1}{2}\left[\left(\frac{b+p_{2} d_{2}}{1+p_{2}}\right)^{2}\right.} \\
& \left.-\left(\frac{b+p_{1} d_{1}}{1+p_{1}}\right)^{2}\right]+\left(\delta_{c 2}-\delta_{c 1}\right) .
\end{aligned}
$$

where the subscripts 1 and 2 refer to TE number 1 and TE number 2 .

The first term in brackets represents the variation in $\delta_{t}$ due to differences between the in-phase components of the two TE's, and includes the variation in $\delta_{t}$ due to the difference in loading between the TE's because of nonequal heater resistances. The term in the second set of square brackets represents the variation in $\delta_{t}$ due to the difference in phase angle between the two TE's. The last term in parentheses represents the variation in $\delta_{t}$ due to differences in current converter characteristics.

The variations in the ac-dc difference due to loading and phase angle differences between TE 1 and TE 2 are presented in Appendixes A and B, respectively. As long as reasonable care is taken to match the two TE's so that they meet the criteria noted in the appendixes these contributions to the variation in ac-dc difference in (6) may be ignored and we can write the variation in ac-dc difference between TE 1 and TE 2 as

$$
\delta_{t 2}-\delta_{t 1}=\frac{a+p_{a} c_{2}}{\left(1+p_{a}\right)}-\frac{a+p_{a} c_{1}}{\left(1+p_{a}\right)}+\left(\delta_{c 2}-\delta_{c_{1}}\right)
$$

which reduces to

$$
\delta_{t_{2}}-\delta_{t_{1}}=\frac{p_{a}}{1+p_{a}}\left(c_{2}-c_{1}\right)+\left(\delta_{c_{2}}-\delta_{c_{1}}\right) .
$$

Noting that $p /(1+p)=R_{h} / R_{t}$,

$$
\delta_{t_{2}}-\delta_{t_{1}}=\frac{R_{h}}{R_{t}}\left(c_{2}-c_{1}\right)+\left(\delta_{c_{2}}-\delta_{c_{1}}\right)
$$

where $c_{2}$ and $c_{1}$ are the ac-dc differences $\left(\delta_{h_{2}}\right.$ and $\left.\delta_{h_{1}}\right)$ due to the change in resistance of the TE, so that

$$
\delta_{t_{2}}-\delta_{t_{1}}=\frac{R_{h}}{R_{t}}\left(\delta_{h_{2}}-\delta_{h_{1}}\right)+\left(\delta_{c_{2}}-\delta_{c_{1}}\right) .
$$

Since $\delta_{c}=\delta_{v}-\delta_{h}$, where $\delta_{v}$ is the ac-dc difference of the TE as a voltage converter, $\delta_{h}$ is the contribution to the ac-dc difference due to the change in heater resistance, and $\delta_{c}$ is the ac-dc difference of the TE as a current converter, then

$\delta_{t_{2}}-\delta_{t_{1}}=\frac{R_{h}}{R_{t}}\left[\left(\delta_{r_{2}}-\delta_{c_{2}}\right)-\left(\delta_{v_{1}}-\delta_{c_{1}}\right)\right]+\left(\delta_{c_{2}}-\delta_{c_{1}}\right)$.

Using $R_{t}-R_{h}=R_{s}$, we may finally write

$$
\delta_{t_{2}}-\delta_{t_{1}}=\frac{R_{h}}{R_{t}}\left(\delta_{v_{2}}-\delta_{v_{1}}\right)+\frac{R_{s}}{R_{t}}\left(\delta_{c 2}-\delta_{c_{1}}\right) .
$$

This is a particularly useful expression since the $\delta_{v}$ 's and $\delta_{c}$ 's for both TE 1 and TE 2 can be obtained by direct measurement.

\section{Thermoelement Replacement Procedures}

To properly use (7), one must know the ac-dc differences of both the old and new TE's as voltage and current converters. Several procedures exist for determining these quantities; the procedure developed at NBS for replacement of working standards is outlined below.

The ac-dc difference of a TVC depends upon the combination of its ac-dc differences as a voltage and current converter. As Fig. 2 shows, the contribution made by current effects begins to dominate at relatively low voltages. In fact, for a typical coaxial TVC set, 90 percent of the ac-dc difference is due to current effects on the second 


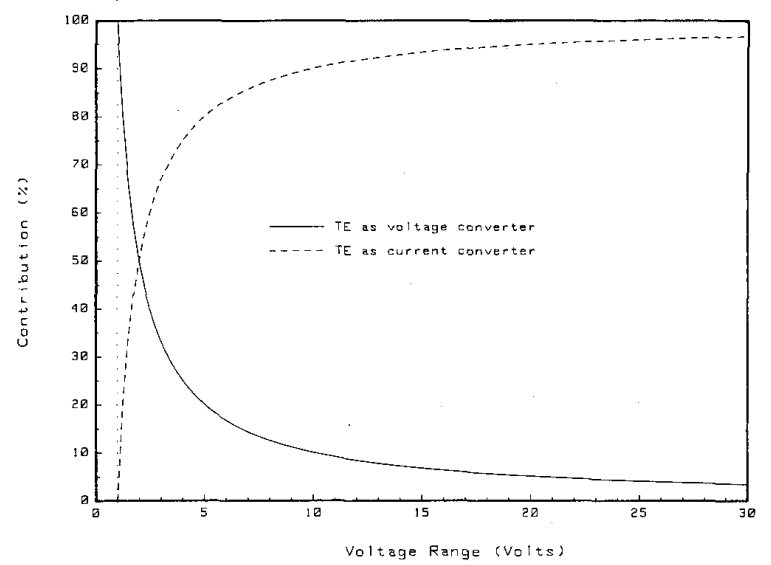

Fig. 2. Relative contribution of the thermoelement to the ac-dc difference of a coaxial TVC as a voltage converter and as a current converter. This data is for a $2.5-\mathrm{mA}$ TE module with a nominal $400-\Omega$ input impedance.

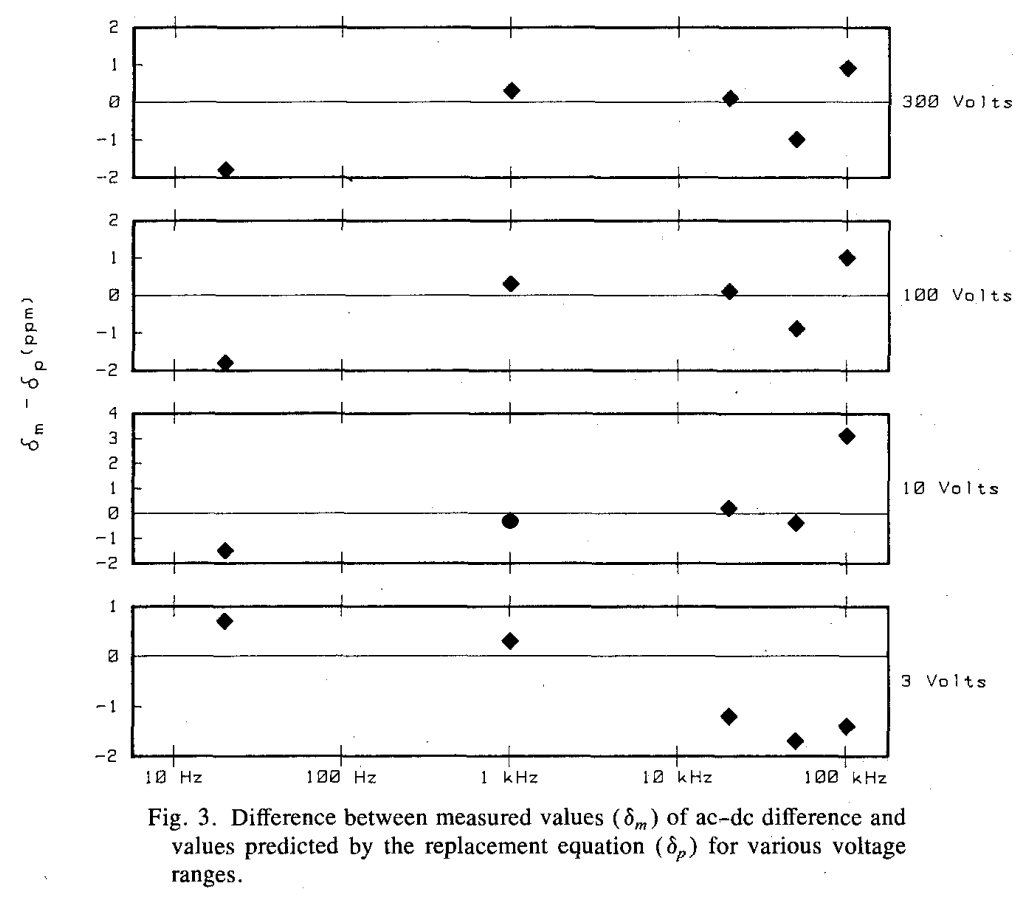

lowest range where a series resistor is used, and the contribution increases for greater voltages. Although thermoelements are generally excellent current converters with ac-dc differences of only a few parts per million at frequencies of up to $1 \mathrm{MHz}$, a new TE should, nevertheless, be measured as a current converter before attempting to apply the replacement equation.

The following is the procedure used at NBS for TE replacement.

1) The phase angle of the candidate replacement TE is checked using, for example, a commercial impedance analyzer. The phase angle must match that of the TE it will replace to within $1 \mathrm{mrad}$ to ensure that the phase angle term in (6) can be neglected.
2) The resistance of the new TE is measured to ensure that loading effects on the series resistor are negligible and the loading term in (6) may be ignored.

3) Intercomparisons are made between the new TE and a TE whose characteristics are known as voltage converters at the lowest voltage range with no series resistor.

4) Intercomparisons are made as a current converter against a TE whose value is known using two matched series resistors [1], [4]. The value of these resistors is chosen to be large enough to test the TE's as current converters, but small enough to avoid high-voltage effects.

5) After obtaining the ac-dc differences of the new TE as a voltage and current converter, the values obtained in steps 3) and 4) are inserted in (7) and the ac-dc difference 
for each voltage range associated with that TE is calculated.

It is extremely helpful to have on hand new or replacement TE's, mounted and characterized in terms of existing TE's. Aside from the obvious advantage of redundancy in the case of TE failure, the measurements required as voltage and current converters are made much easier if a TE of the same current rating is available as a check standard. If such a TE is unavailable, a TE of a different current rating may be used, but the measurements are more difficult, and current level dependencies must be considered. If a TE of a different current rating is used as a standard, its ac-dc difference must be known at the different current rating, both as a current converter and as a voltage converter.

\section{Results}

Tests of the replacement technique were performed with various TVC ranges configured using two different thermoelement modules. AC-DC differences for the different voltage ranges employing both TE's were determined by comparison with a separate standard TVC. The variation between the measured values, $\delta_{m}$, and predicted values, $\delta_{p}$, using (7) for one pair of TE's is shown in Fig. 3. Tests were made on the $3-, 10-, 100-$, and $300-\mathrm{V}$ ranges at frequencies of $20 \mathrm{~Hz}, 1,20,50$, and $100 \mathrm{kHz}$. The standardpattern TE's used in this test had $400-\Omega$ heater resistances with current ratings of $2.5 \mathrm{~mA}$. The ac-dc differences of the test TE's differed by as much as $16 \mathrm{ppm}$ as voltage converters and $2 \mathrm{ppm}$ as current converters. Small ac-dc differences for current converters, such as this, are common for good-quality TE's. Agreement between the measured and predicted values using this method is about 3 ppm or better at the test points given above. This performance is quite satisfactory for essentially all routine applications.

Similar tests were carried out to check for any additive effect present in the iterative use of this procedure. No additive effect was found to be present within the accuracy of the comparator system.

To test for loading effects on a series resistor, a TE module was fabricated with an easily replaceable resistor in series with the heater. The value of this resistor was increased in steps of approximately $5 \Omega$ from 408 to 428 $\Omega$. Again, no significant variation was found.

An informal survey of commercial TVC's was undertaken to ensure that loading effects and phase angle corrections could be neglected in commercial coaxial TVC sets. The phase angles and resistances for the TE's in these sets were checked using an impedance analyzer. All TE's measured, from different manufacturers as well as NBS, had heater resistance values within $5 \Omega$ of nominal, and phase angle variations of less than $1 \mathrm{mrad}$. The replacement procedure should, therefore, be perfectly suitable for commercially made coaxial TVC sets, as well as the NBS working standards.

\section{CONCLUSION}

A procedure to predict the ac-dc difference of coaxial TVC's after one TE has been replaced with another has been developed at NBS. Results show that for routine applications using working standards, the procedure predicts the ac-dc difference well enough to be placed into normal use. This procedure saves a considerable amount of time and effort when a TE is damaged or fails.

APPENDIX A

Contributions Due to Variations in Loading

To consider the effects due to variations in loading on the series resistor when a TE of one heater resistance is replaced by a TE of slightly different heater resistance, we write the variation in the in-phase term in (6) as

$$
\Delta_{\lambda}=a\left[\frac{1}{1+p_{2}}-\frac{1}{1+p_{1}}\right]+\left[\frac{p_{2} c_{2}}{1+p_{2}}-\frac{p_{1} c_{1}}{1+p_{1}}\right]
$$

where $\Delta_{\lambda}$ is the variation in ac-dc difference due to replacing one TE with another of different resistance. The first term in (8) may be written

$$
\Delta_{\lambda}^{\prime}=a \frac{\left(1+p_{1}-1-p_{2}\right)}{\left(1+p_{2}\right)\left(1+p_{1}\right)} \simeq a \frac{\left(p_{1}-p_{2}\right)}{\left(1+p_{a}\right)^{2}}
$$

where $p_{a}$ is the average value of $p_{1}$ and $p_{2}$. This equation inay be used to predict the variation in ac-dc difference due to loading expressed as a percentage of $a$. For example, if the loading effect is to be kept to less than 1 percent of $a$, generally a negligible fraction of the ac-dc difference, for $p_{a}=1$ (as a worst-case analysis), then ( $p_{1}$ $-p_{2}$ ) must be 4 percent or less, or about $16 \Omega$ for a typical 400- $\Omega$ TE module. This is not a difficult criterion to meet in practice. Fig. 4 shows calculated variations in acdc difference for changes in TE module resistance for $R_{h}$ $=400 \Omega$ and for several values of series resistance. The calculated variations for larger values of series resistance are even less.

To treat the second term in (8), we first define $q_{1}=$ $p_{1} /\left(1+p_{1}\right), q_{2}=p_{2} /\left(1+p_{2}\right)$, and $q_{a}$ as the average value of $q_{1}$ and $q_{2}$ so that $q_{1}=q_{a}(1-\alpha)$ and $q_{2}=q_{a}(1$ $+\alpha$ ), where $2 \alpha$ is the difference between $q_{1}$ and $q_{2}$. Likewise, we define $c_{a}$ as the average value of $c_{1}$ and $c_{2}$ so that $c_{1}=c_{a}(1-\beta)$ and $c_{2}=c_{a}(1+\beta)$. The second term in (8) is then

$$
\begin{aligned}
q_{2} c_{2}-q_{1} c_{1}= & c_{a} q_{a}(1+\alpha)(1+\beta) \\
& -c_{a} q_{a}(1-\alpha)(1-\beta)
\end{aligned}
$$

which reduces to

$$
2 c_{a} q_{a}(\alpha+\beta)
$$

The contribution to the ac-dc difference associated with loading variations in the second part of (8) is $\Delta_{\lambda}^{\prime \prime}=$ 


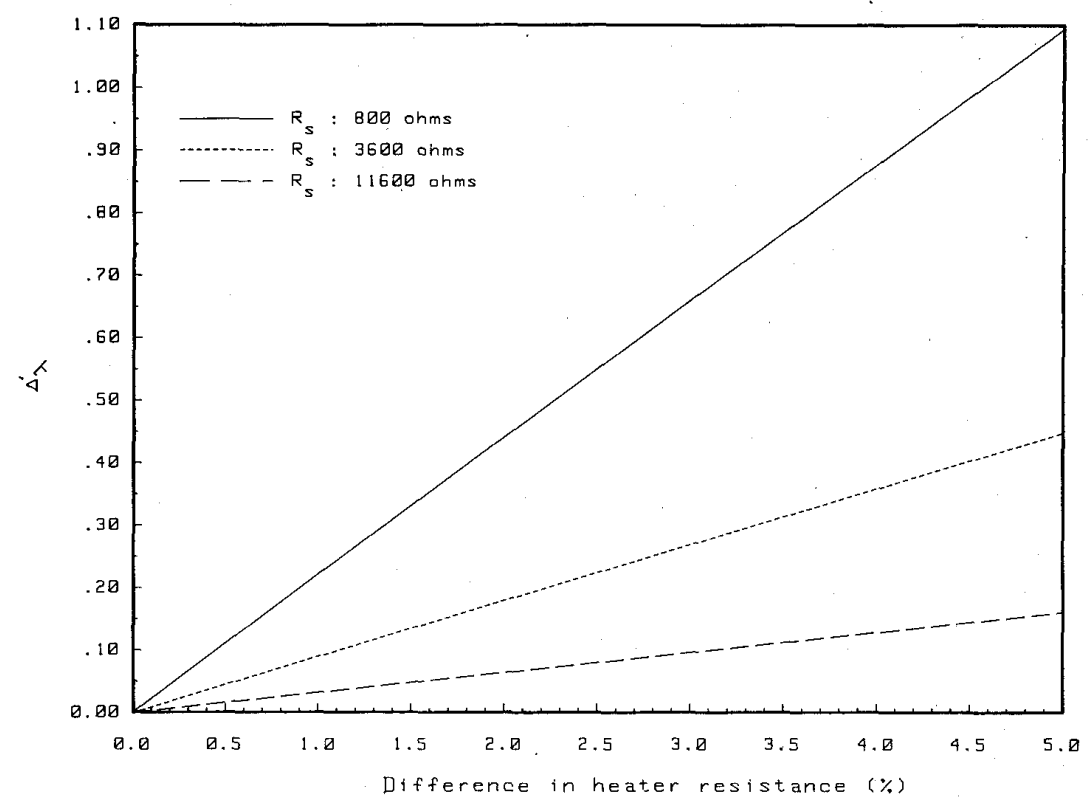

Fig. 4. Calculated contribution to ac-dc difference from $\Delta_{\lambda}^{\prime}$, as a percentage of $a$, due to variations in the resistance of the TE module for various values of the series resistor, $R_{s}$.

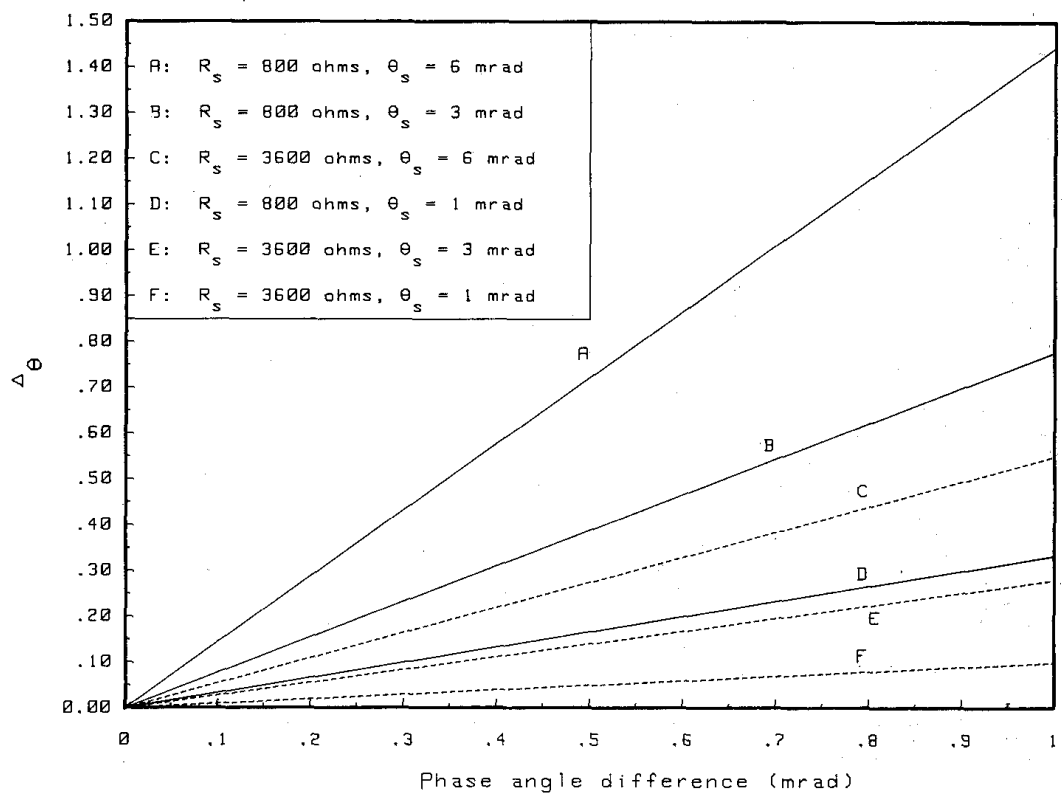

Fig. 5. Calculated contribution, in parts per million, to ac-dc difference due to variations in the phase angle of the TE module for several values of series resistance, $R_{s}$, and resistor module phase angles, ${ }_{s}$.

$2 c_{a} q_{a} \alpha$. Since

$$
\alpha=\frac{q_{2}-q_{1}}{2 q_{a}}
$$

we may write the factor associated with loading as

$$
\Delta_{\lambda}^{\prime \prime}=c_{a}\left(q_{2}-q_{1}\right) \text {. }
$$

For good quality TE's the $c$ term is generally $\leq 10 \mathrm{ppm}$ at audio-frequency and $\leq 50 \mathrm{ppm}$ at $100 \mathrm{kHz}$. Using this $100 \mathrm{kHz}$ value for $c_{a}$ and taking $p_{1}=1$ as the worst-case, the TE's must be matched to within about 8 percent of nominal heater resistance for the variation in ac-dc difference arising from a change in loading in the second term in (8) to be less than $1 \mathrm{ppm}$. For typical $400-\Omega$ heat- 
ers, this is $32 \Omega$. Therefore, if the TE's are matched sufficiently well to disregard the first term in (8), the second term may be ignored as well.

Since the contributions to the total ac-dc difference due to loading variations in the terms of (8) are likely to be negligible for routine applications with commonly available TE's, the final expression for TE replacement is derived in the main text with the assumption that the loading is unchanged.

\section{APPENDIX B}

Phase Angle Contributions

The variation in ac-dc difference from the phase angle contribution becomes important only when the difference in phase angle from one TE to another becomes large coupled with a large phase angle for the series resistor. The variation in ac-dc difference due to nonequal phase angles, $\Delta_{\theta}$, in the phase angle term from (6) is

$$
\Delta_{\theta}=\frac{1}{2}\left[\left(\frac{b+p_{2} d_{2}}{1+p_{2}}\right)^{2}-\left(\frac{b+p_{1} d_{1}}{1+p_{1}}\right)^{2}\right] .
$$

Squaring the terms in brackets, and collecting like terms

$$
\Delta_{\theta}=\frac{p}{1+p}\left[\left(d_{2}-d_{1}\right) \frac{b}{1+p}+\frac{p}{1+p} \frac{\left(d_{2}+d_{1}\right)}{2}\right] .
$$

where $p_{1}=p_{2}=p$, and the differences in loading are not considered.

Letting $d_{a}=\left(d_{2}+d_{1}\right) / 2$ the average value of $d$

$$
\Delta_{\theta}=\frac{p\left(d_{2}-d_{1}\right)\left(b+p d_{a}\right)}{(1+p)^{2}}
$$

If $b \simeq \theta_{s}$, the phase angle of the transimpedance of the series resistor, $d_{a} \simeq \theta_{h}$, the average phase angles of the thermoelements, and $\Delta \theta_{h}=\left(d_{2}-d_{1}\right)$, then

$$
\Delta_{\theta}=\frac{R_{h}}{R_{t}} \Delta \theta_{h}\left[\frac{R_{s}}{R_{t}} \theta_{s}+\frac{R_{h}}{R_{t}} \theta_{h}\right] .
$$

The greatest phase angle contribution due to the difference in the TE's will occur at low voltage ranges where $R_{h}$ is a significant fraction of $R_{t}$. Fig. 5 shows the calculated variations in ac-dc difference for varying TE module phase angles and several values of series resistance and resistor module phase angles for a nominal 400- $\Omega \mathrm{TE}$ module and $\theta_{h}=1 \mathrm{mrad}$. For the worst case, where $R_{h}$ $=R_{s}$, if the phase angle contribution is to be less than 1 $\mathrm{ppm}$, the phase angles of the TE's must be matched to within $1 \mathrm{mrad}$ for an average phase angle $\left(\theta_{h}\right)$ of $1 \mathrm{mrad}$. Since this is also not a difficult criterion to meet, the second bracketed term in (6) will be omitted from the derivation of the final expression for overall ac-dc difference with a replacement TE.

\section{REFERENCES}

[1] F. L. Hermach, "An investigation of the uncertainties of the NBS thermal voltage and current converters," Nat. Bur. Stand, U.S., Rep. NBSIR 84-2903, Apr. 1985

[2] B. D. Inglis, "Evaluation of the ac-dc transfer errors for thermal converter-multiplier combinations," Metrologia, vol. 16, pp. 177-181, 1980.

[3] F. L. Hermach and E. S. Williams, "Thermal converters for audiofrequency voltage measurements of high accuracy," IEEE Trans. Instrum. Meas., vol. IM-15, pp. 260-268, Dec. 1966.

[4] E. S. Williams, "Thermal current converters for accurate ac current measurements," IEEE Trans. Instrum. Meas., vol. IM-25, pp. 519523, Dec. 1976 